\title{
ASEGURAMIENTO DE CALIDAD EN GESTIÓN DE INSTITUCIONES DE EDUCACIÓN SUPERIOR: EXPECTATIVAS Y DESAFÍOS
}

\section{Introducción}

El proceso piloto de acreditación institucional, recientemente desarrollado por la Comisión Nacional de Acreditación (CNAP), ha dejado interesantes lecciones. Algunas de ellas se recogen en el estudio sobre modelos de gestión en la educación superior en Chile desarrollado por Raúl Atria ${ }^{i}$, sobre la base de los informes de autoevaluación de algunas universidades integrantes del Consejo de Rectores acogidas a la iniciativa. El estudio ofrece una oportunidad para reflexionar sobre las expectativas y desafíos que pueden vislumbrarse, en miras al fortalecimiento de nuestro sistema de educación superior. ¿Qué cabe esperar del establecimiento definitivo de un modelo de aseguramiento de la calidad como el ensayado? Las lecciones que pueden extraerse de la experiencia piloto aportan antecedentes útiles para apreciar su impacto en la gestión de las propias instituciones acreditadas, también sobre los desafíos que deberán enfrentar los futuros procesos de acreditación y las definiciones que ello demanda en las políticas públicas sobre educación superior.

Sobre las instituciones pesa, ante todo, el desafío de incorporar los beneficios directos de la experiencia a sus propios sistemas de gestión. Y no solamente porque al cabo del plazo de vigencia del acuerdo de acreditación deberán responder de los avances alcanzados, sino que, también y principalmente, porque la experiencia cobrará todo su valor en la medida del aprendizaje

i Ver en este mismo número: Atria, Raúl. "La gestión de las universidades del Consejo de Rectores". 
obtenido. Los futuros procesos de acreditación podrán verse también enriquecidos con la información acumulada en la reciente experiencia. En especial, al observarse algunos nudos críticos que afectan, de manera más o menos común, la gestión de las instituciones, y que plantearán desafíos importantes para éstas y para los evaluadores.

Desde el campo de las políticas públicas, por último, cobra especial relevancia la necesidad de avanzar en algunas definiciones que subyacen en la discusión sobre el modelo de aseguramiento de la calidad que se pretende instalar, y corregir, de paso, ciertas restricciones que lo afectan.

\section{Desafíos para las instituciones acreditadas}

Someterse a una acreditación ante un organismo externo resultó para estas instituciones un desafío importante. Además de emprender un estudio de evaluación interna, de suyo complejo y exigente, estas organizaciones se vieron enfrentadas a la necesidad de dar cuenta de la consistencia de sus propósitos, políticas y mecanismos que aseguran su calidad ante un comité de observadores externos. Con distintos grados de éxito, probablemente medidos en función de las expectativas y el resultado -en términos del número de años por el que se extiende la acreditación obtenida-, las instituciones que la alcanzaron fueron, en su oportunidad, capaces de demostrar que cuentan con tales mecanismos con algún grado razonable de desarrollo y poseen, además, la capacidad para reconocer sus falencias y tomar las decisiones necesarias para superarlas. Estas capacidades, con certeza, serán puestas a prueba en los años venideros. Lo están siendo ya, porque el tiempo transcurre inexorablemente. El estatus de institución acreditada, cualquiera sea su valor, tiene fecha de caducidad y, al cabo, el trance deberá ser vivido nuevamente. En eso consiste precisamente el juego.

Pero, entretanto, habrán de incorporarse al análisis elementos adicionales. Por una parte, los procedimientos, los criterios y la propia 
experiencia de los evaluadores externos se irán perfeccionando en la medida de la frecuencia de nuevas acreditaciones. Este perfeccionamiento importará una mayor capacidad auditora: una sensibilidad más fina para percibir las fisuras y vacíos que se acusan en la línea de consistencia entre propósitos, acciones y resultados. $\mathrm{Si}$, en una primera mirada, propósitos razonablemente formulados, instrumentos de política y capacidad de gestión pudieron resultar suficientes para reconocer que una institución "se encuentra en camino" de mejoramiento, una evaluación posterior habrá necesariamente de preguntarse qué tanto se ha avanzado en esa dirección. ¿Qué tan bien han funcionado los mecanismos descritos y observados? ¿Qué tanto han mejorado las capacidades institucionales ya evaluadas? Y esta segunda mirada podría ser no necesariamente más estricta pero, indudablemente, sí más certera.

Por otro lado, hay nuevos desafíos que enfrentar en un contexto institucional y de mercado en plena evolución. En cuatro o seis años son muchos los nuevos factores, tanto internos como externos, que entrarán a jugar: fusión y expansión territorial de instituciones, incorporación de proveedores foráneos, cambios en la base socioeconómica de los estudiantes, restricción al acceso a campos clínicos, universidades corporativas, nuevos formatos para la oferta educativa y tantos más que desde ya pueden vislumbrarse. Nuevamente las capacidades institucionales serán puestas a prueba, pero esta vez la pregunta será: ¿están siendo enfrentados estos nuevos desafíos con la operación de los mecanismos de aseguramiento descritos en el proceso de acreditación? Más aún, ¿qué tanto las capacidades alcanzadas expresan una mejor posición de la institución para enfrentar los desafíos de la nueva coyuntura?

Si pensamos que la acreditación es, en sí misma, un instrumento de mejoramiento institucional, lo esperable sería que la institución acreditada se vea fortalecida y que dicha fortaleza opere justamente ahí donde puede desplegar todo su beneficio. Podría objetarse que la acreditación solamente da cuenta del estado de una institución, pero que no la mejora en sí misma. Que el 
aseguramiento de la calidad se justifica tan sólo por la garantía a la fe pública depositada en ella para otorgar títulos y grados reconocidos. Pero, aún en tal caso, las mismas interrogantes se trasladan de las instituciones al sistema. Tarde o temprano, alguien se preguntará: y al fin, ¿qué logramos con todo esto? Como quiera que se enfrente el asunto, la pregunta debe ser afrontada desde ya. La respuesta pertinente, al final del camino, se construirá con lo hecho en el trayecto.

\section{Contribución de la lógica evaluativa}

Una cosa es clara: el proceso de acreditación ha incorporado una cierta lógica de razonamiento que no se encontraba del todo asentada en la gestión de muchas de nuestras instituciones de educación superior. Esta lógica involucra un desplazamiento, en la fundamentación de los juicios de calidad, desde la capacidad argumentativa a la evidencia, desde el juicio de valor al de consistencia, desde el énfasis en los insumos a la pregunta por los resultados. Y esto no es menor. El supuesto básico de la acreditación institucional, como mecanismo de aseguramiento de la calidad, es la afirmación de que ella promueve un proceso de mejora continua en las instituciones. Esta expectativa resultaría muy débil de cifrarse sólo en el efecto de un estímulo por cierta mejora general de una institución para ostentar la condición de "acreditada" y, por ello, de verse fortalecida su imagen pública (junto con la expectativa de acceso a condiciones de financiamiento institucional o estudiantil, que todavía resulta poco relevante y que, aun cuando constituya un incentivo, no representa un beneficio en sí mismo). Su fortaleza radica, también -y muy especialmente- en la expectativa de que la lógica con que opera el juicio evaluativo promueva el desarrollo de prácticas de gestión aptas para lograr mejoras efectivas y perceptibles. Es decir, que promuevan un efectivo empowerment de las instituciones, expresado en la instalación de una capacidad adicional que les permita avanzar de modo más directo hacia la concreción de sus propósitos. 
En efecto, la lógica evaluativa del proceso de acreditación, al menos en los términos como se ha dado en la experiencia observada, responde a un modelo de análisis susceptible de ser aplicado al sistema de toma de decisiones en múltiples dimensiones de la actividad institucional, asegurando resultados más predecibles a las mismas, haciéndolas más efectivas. La secuencia de atributos, que constituye la base de aquellas preguntas que deben ser respondidas en una evaluación interna o externa, representa el instrumento de gestión que haría operar tal mejoramiento continuo. Tal es la expectativa "fuerte" del modelo. La capacidad de incorporar este método de planificación estratégica a la realidad cotidiana de cada institución se constituye así en una oportunidad. El desafío puede traducirse en la instalación de una core competence en los sistemas de dirección y planificación, tanto en el ámbito general como en las respectivas unidades académicas.

\section{Nudos críticos para la evaluación}

Para los mecanismos de aseguramiento y, en particular, para el desarrollo de las instancias de evaluación externa, se presentan igualmente desafíos importantes. Poniendo atención al estado de estas variables en el modelo evaluativo descrito, según se observa en el estudio realizado por Raúl Atria, es posible destacar algunos aspectos que se perfilan como nudos críticos para la evaluación institucional de las universidades observadas en dicho estudio. Pero no solamente de ellas, dado que es perceptible que muchos de estos nudos -no todos, por supuesto- resultan comunes a la generalidad de instituciones no incluidas en el análisis.

a) El asunto de los propósitos. En el plano de la cautela de la misión, y en un contexto de cierta fragilidad en la difusión de la estrategia en la comunidad universitaria, se acusa en el estudio un problema relativo a la vigencia, socialización y efectividad de los propósitos, más allá de "cierta comunidad de lenguaje y de discurso". En una primera mirada, esta pareciera ser una variable de mayor complejidad en las universidades tradicionales o públicas que en 
las entidades privadas nuevas. Sin embargo, una observación más profunda muestra un problema común a todas las instituciones. Necesariamente, para que una declaración de misión resulte operativa exige definiciones que importan cierta preeminencia de determinadas áreas de actividad por sobre otras. Es aquí donde la comunidad de discurso entra en choque. Si se logra una definición de propósitos ampliamente compartida, ello afectará a la operatividad de la declaración, puesto que de algún modo forzará a renunciar a aquellas definiciones que importan afirmar dichas preeminencias. Por el contrario, cuanto más claras y dispositivas resulten tales definiciones, menor comunidad de discurso, al menos entre aquellas unidades e integrantes de la organización académica que vean en algún grado afectados sus propósitos e intereses específicos. Esta tensión no tiene por qué resultar más ni menos intensa en una u otra categoría institucional. Más bien, pareciera tener relación con la complejidad y trayectoria de la organización. Para las instituciones más cercanas a los cánones centrales de una definición tradicional de universidad pública, la cuestión estriba en dirimir cuáles de sus funciones clásicas adquirirán primacía y en qué grado. Para las instituciones periféricas, menos comprometidas con dichos cánones, el equilibrio deberá buscarse entre lo propio y singular frente a lo que constituya parte del ethos reconocido como una genuina experiencia de educación superior. Universidades que se definen como puramente docentes, o que ensayan una caracterización asociada a mercados geográficos o socioeconómicos -y particularmente, las entidades del círculo periférico más externo, como institutos profesionales y centros de formación técnica-, deberán resolver esta última ecuación. Al fin y al cabo, todas las instituciones requieren acotar cierta singularidad de propósitos para caracterizar su propia identidad, cuestión que el evaluador deberá sopesar al calificar la consistencia y vigencia de su formulación.

b) Información institucional. La producción, circulación y uso de la información institucional se presenta también como una cuestión transversal. Mucho tiene que ver con el desarrollo las 
capacidades de gestión, aludidas antes. Sea porque la motivación preponderante haya respondido a exigencias externas o por la conveniencia de consolidar sistemas de toma de decisiones en función de una mayor eficiencia de procesos y empleo de insumos, pareciera que en el último tiempo se han alcanzado avances significativos en este campo. Pero, igualmente, se vislumbra la dificultad de superar el primer nivel del proceso. En decir, mucha información pero poco uso efectivo de la misma. La insuficiencia de los sistemas de accesibilidad no privilegiada a la información, mediante sistemas informáticos, parece ser un síntoma de ello. Finalmente, excesiva información puede producir tanta oscuridad como su ausencia. La selección de datos relevantes y su traducción en indicadores de desempeño comúnmente aceptados es una cuestión de suyo compleja. Afecta intereses, exige importantes esfuerzos de análisis y síntesis, y requiere siempre conjurar los habituales fantasmas del reduccionismo y la hipersegmentación. Porque ni todo es tan simple ni cada caso tan especial. Pero hay todavía logros importantes que alcanzar en la obtención de cierta información relevante. En particular, todo lo relacionado con la percepción del entorno, la evaluación de los resultados, el seguimiento de los egresados y la retroalimentación desde la industria. Qué tan representativa pueda ser la medición de estos contextos es todavía una cuestión pendiente.

c) Recursos humanos. Las dificultades para la formación de una masa crítica de académicos, mediante políticas estables y eficientes para su reclutamiento, actualización y desvinculación, es otro tema común. El nudo crítico radica aquí en la evaluación de desempeño, que, a la luz de las conclusiones del informe, descansa más en asuntos de forma que de sustancia. Medir la productividad de los académicos en investigación relevante es un problema conocido. Pero, aun si se la excluye del análisis, la gestión de la productividad en el ámbito exclusivamente docente es, en sí misma, un tema angular, tanto más crítico como menos resuelto. No sólo por la necesidad de armonizar efectividad en el rendimiento estudiantil y libertad de cátedra. Por cierto que medir la efectividad docente 
requiere cambios importantes en el ethos de la comunidad académica. Pero, también, importa la necesidad de considerar nuevos modelos evaluativos, incorporando elementos de juicio que permitan asociar el desempeño docente a los resultados de la experiencia formativa, en términos de aprendizajes esperados y logrados. Ello exige anticipar el producto de un proceso de larga duración y despejar su dependencia de otras variables, como las aptitudes de los alumnos o las condiciones de contexto. También supone prever externalidades que se generan en distintas direcciones. Se trata, en definitiva, de un aspecto de la gestión institucional que ofrece un importante espacio a la innovación. La exposición a una evaluación externa debería constituir, más que una limitación, una motivación adicional para una mayor apertura de este espacio, planteando un interesante desafío, tanto para las propias instituciones como para las instancias de evaluación.

d) Provisión de programas. La innovación curricular aparece como un fenómeno fuertemente instalado, pero pocas instituciones han logrado saltar del discurso a la traducción de tales innovaciones en un mejoramiento efectivo en la formación de los estudiantes. Ello se liga, además, con la escasa validez en la desescolarización del aprendizaje. El proceso de Boloña ha tenido una influencia significativa en este fenómeno, en particular, la recurrente referencia a un enfoque basado en competencias. Pero siguen pendientes algunas interrogantes sustantivas que plantea este enfoque: la compatibilidad entre formación de competencias genéricas y específicas; el equilibrio entre la amplitud y extensión de los objetivos curriculares con la capacidad de asegurar determinadas habilidades esenciales, y, especialmente, la armonización de estrategias sistémicas para introducir prácticas metodológicas más efectivas de diseño instruccional -un elemento inseparable del modelo- frente a la llamada "autonomía hacia adentro", con particular acento en la libertad académica.

e) Estructura organizacional. Hay también otros nudos críticos que afectan de modo muy diferente según las categorías 
institucionales de que se trate. Ellos tienen que ver, fundamentalmente, con su estructura organizacional. Problemas como la participación segmentada por estamentos, la insuficiente "ciudadanía participativa" y su expresión en procesos participativos transversales, la combinación entre estructuras jerárquicas y planas, así como la construcción de liderazgos internos, parecieran ser más atingentes a cierta clase de instituciones y no necesariamente extrapolables a todas las entidades del sistema. Asumir esta distinción será también un desafío importante para los evaluadores externos. Además, calificar tales cuestiones importa, frente a las instituciones "periféricas", un especial esfuerzo para no incorporar juicios de valor que puedan contaminar una ponderación de la consistencia interna del modelo evaluado. Ello no significa desconocer la validez de tales postulados en el contexto de las instituciones llamadas tradicionales o de aquellas que, al menos en su declaración de propósitos, proclaman la adhesión a los valores inherentes al paradigma de una universidad pública latinoamericana. Significa, más bien, que muchas instituciones responden a una dinámica organizacional basada en postulados enteramente distintos y, por lo mismo, reclaman un estatus de legitimidad que no puede ser discutido a priori. Con todo, tampoco se puede concluir que cualquier modelo organizacional deba ser aceptado de plano. Por cierto que la estructura de los cuerpos directivos y los sistemas de toma de decisiones deben responder a criterios de transparencia, eficiencia y eficacia. La viabilidad de la institución como tal, tanto como su capacidad de responder cabalmente a los compromisos asumidos con los destinatarios de sus servicios, exigirá, en más de un caso, observar atentamente prácticas de gestión y modelos organizacionales que garanticen la seriedad y efectividad de sus propósitos declarados.

\section{Desafíos en políticas públicas}

Una tercera perspectiva de este comentario apunta a las políticas públicas. Este es el campo principal en que se desenvuelven algunas 
de las conclusiones del informe citado, relativas en especial a la tensión entre autonomía "hacia fuera" y responsabilidad. ¿Cuáles son los desafíos que, al respecto, se presentan para las instancias cautelares de interés público? Esta pregunta apunta a la definición de políticas públicas en función de un adecuado establecimiento de un sistema nacional de aseguramiento de la calidad. Dentro de ellas, el desarrollo de un marco legal apropiado es, obviamente, una condición necesaria aunque no suficiente. Existe en la actualidad un régimen regulatorio, consagrado en la Ley Orgánica Constitucional de Enseñanza (LOCE), que aporta elementos significativos. Como es sabido, está pendiente además, en último trámite legislativo, un proyecto de ley que tiene por objeto, precisamente, establecer un sistema nacional de aseguramiento de la calidad. Sin entrar a su análisis, es posible formular desde ya algunos desafíos que, en parte por vía legislativa y en parte administrativa, deberán ser asumidos en algún momento y que conviene tener presentes.

El primer desafío consiste en definir si la aproximación al aseguramiento de la calidad de las instituciones autónomas constituye un instrumento de fomento o de control. En la actualidad, con sus aciertos y debilidades, nuestro país cuenta con un marco regulatorio dotado de importantes mecanismos de control ex ante para la incorporación de instituciones al sistema, mediante el proceso, primero, de reconocimiento oficial de las instituciones nuevas y, luego, de su acreditación (licenciamiento) ante el Consejo Superior de Educación o el Ministerio de Educación, según el caso, como condición para su continuidad y plena autonomía. Así las cosas, ¿Es el aseguramiento de la calidad y, en particular, la nueva acreditación -institucional o de programas- un mecanismo de control ex post, aplicable una vez que las instituciones han alcanzado su autonomía plena? ¿Se trata, por el contrario, de un instrumento de fomento, previsto para alcanzar un mejoramiento general de la calidad de nuestro sistema, desde un fortalecimiento de las capacidades institucionales de las organizaciones que lo constituyen? 
En el primer caso, la acreditación y los restantes mecanismos asociados deberían ser obligatorios, con mayor o menor explicitación de tal exigencia; pero, en todo caso, como condición necesaria para la supervivencia de todas las instituciones. Esta opción presenta un riesgo, ya evidenciado en las fórmulas de control ex ante que han operado hasta la fecha, respecto del cual se ha observado que "la obligatoriedad implica habitualmente el desarrollo de una actitud de obediencia que con dificultad genera aceptación y adhesión". En el segundo, la experiencia debería ser claramente voluntaria, sin perjuicio de los incentivos que se diseñen para promoverla. Esta disyuntiva subyace en el extenso debate que ha acompañado al proyecto de ley en actual tramitación. Las argumentaciones se suceden invocando alternativamente uno $\mathrm{u}$ otro enfoque. Pero el desafío principal estriba en superar la retórica y dilucidar la cuestión desde ya. Aunque nos pese, las capacidades estatales para satisfacer las crecientes necesidades de educación terciaria en nuestros jóvenes y trabajadores es claramente insuficiente. El concurso de la iniciativa privada es, tan sólo por ello y más allá de otras consideraciones, simplemente ineludible. La oportunidad de promover buenas prácticas, desarrollar una mayor profesionalización en la gestión de las instituciones y, especialmente, de congregar en el sector las mejores capacidades nacionales y locales, supone un marco regulatorio que dé garantías de estabilidad y certeza en el mediano y largo plazo. Esta es una consideración básica que inspira las políticas de desarrollo de tantos otros sectores de servicios esenciales, como energía, transporte, telecomunicaciones o seguridad social. ¿No será hora de asumir nuestro sistema de educación superior con igual perspectiva? La pregunta puede también formularse de modo inverso: ¿qué resulta a esta altura más importante? ¿Suprimir las instituciones de "baja calidad" o promover que más y mejores instituciones presten un servicio de "buena calidad"? La respuesta a esta interrogante puede ofrecerse desde la misma lógica antes enunciada. El reto principal estriba en hacer consistentes las políticas con los propósitos declarados. 
Un segundo desafío se desprende de la misma pregunta. La experiencia piloto de acreditación institucional ha contribuido a despejar ciertos supuestos que sustentan algunos instrumentos de política pública, en actual vigencia, que resulta ineludible traer a colación. En efecto, subsiste en el sistema, tanto en el plano legislativo como en el de las decisiones administrativas, un criterio discriminatorio para el tratamiento de ciertas categorías institucionales. Ello se manifiesta en el acceso al Aporte Fiscal Directo (AFD), a fondos concursables de desarrollo institucional -en particular el caso del programa MECESUP- y a mecanismos desiguales de financiamiento estudiantil. Tal discriminación no obedece a una lógica congruente con el aseguramiento de la calidad ni con otra política pública diferente, al menos conocida. En el caso del financiamiento estudiantil y del AFD, podría atribuirse a razones históricas. Pero el caso del MECESUP no admite siquiera esta explicación. A falta de ella, sólo es posible inferir que responde a un supuesto de "calidad" a priori, que obvia simplemente los mecanismos de certificación diseñados para tal efecto. No se trata de reivindicar derechos o expectativas. La cuestión estriba en aplicar en el sistema los mismos principios de mejoramiento que se espera instalar en las instituciones: consistencia, transparencia, efectividad, en una palabra: accountability.

\section{Conclusión}

Que tendremos un sistema de aseguramiento de la calidad de instituciones de educación superior autónomas, basado en mecanismos de acreditación, es ya un hecho escasamente controvertible. Más allá de las discusiones sobre las formas que adoptará su consagración jurídica, que aún persisten, pareciera que el programa piloto llevado a cabo por la CNAP, resultando exitoso, ha puesto en marcha un proceso dirigido a su plena instalación. Se trata, por lo demás, de un fenómeno que se extiende en todas partes y, difícilmente, nuestro país podría escapar de su influencia.

Es de esperar que las expectativas que ofrece el modelo se traduzcan en frutos concretos y duraderos, tanto para las propias 
instituciones como para el sistema de educación superior en su conjunto. Entonces, aprovechar las lecciones que deja la experiencia piloto constituye una oportunidad. Entre otros beneficios, este aprendizaje promete traducirse en una mayor profesionalización de la gestión de las instituciones y en un fortalecimiento de las instancias de aplicación de los mecanismos de certificación. Profesionalizar, en este caso, significa desarrollar métodos efectivos y replicables, capaces de traducir en resultados los propósitos perseguidos. También, significa incrementar fuertemente la masa crítica de académicos y profesionales calificados para dirigir eficientemente los procesos propios de la actividad.

A pesar del significativo avance en las últimas décadas, Chile cuenta todavía con una baja tasa de cobertura en su educación superior. Igualmente, es escasa su productividad en investigación relevante. Menor todavía la contribución de sus universidades en la innovación y competitividad de la industria. Mejorar estos aspectos plantea fuertes desafíos, tanto para las instancias cautelares del interés público como para todos los actores institucionales de la educación terciaria. Ello importa asumir que no todas las cosas deben hacerse del mismo modo como hasta ahora. A pesar de la importante diversificación y heterogeneidad de nuestras instituciones, la experiencia muestra numerosos aspectos críticos que resultan bastante comunes. Compartir información, difundir buenas prácticas, establecer alianzas y diseñar fórmulas eficaces de cooperación, articulación y complementación de capacidades, es un camino que no debe ser descuidado. Ello plantea la conveniencia de poner mayor énfasis en fortalecer las confianzas que en destacar las diferencias.

Por conocida, no conviene olvidar otra realidad también evidente: una condición particular del servicio que prestan las instituciones educativas se encuentra en el carácter simbólico, intangible, del producto que entregan a la sociedad. Un grado académico, un título profesional, la certificación de estudios cursados, son valiosos en la medida en que la comunidad les 
reconoce certeza. Más allá de las condiciones formales que contribuyen a esta certeza, su valor se basa en un principio de confianza. Asegurar la calidad significa también robustecer este principio. Para ello, existen ciertas condiciones, ya conocidas, que permiten incrementar este activo social: preservar las tradiciones que nos confieren identidad; educar en la confianza, como virtud y como valor; cumplir los compromisos adquiridos, e incrementar los espacios de expresión de la sociedad civil, fortaleciendo la subsidiariedad. 